\title{
Optimization of Power Allocation for a Multibeam Satellite Communication System with Interbeam Interference
}

\author{
Heng Wang, Aijun Liu, Xiaofei Pan, and Jiong Li \\ College of Communications Engineering, PLA University of Science and Technology, No. 2, Yudao Street, Nanjing 210007, China \\ Correspondence should be addressed to Heng Wang; wangheng0987654321@126.com
}

Received 22 October 2013; Accepted 24 December 2013; Published 16 January 2014

Academic Editor: Hanan Luss

Copyright (C) 2014 Heng Wang et al. This is an open access article distributed under the Creative Commons Attribution License, which permits unrestricted use, distribution, and reproduction in any medium, provided the original work is properly cited.

\begin{abstract}
In multibeam satellite communication systems it is important to improve the utilization efficiency of the power resources, due to the scarcity of satellite power resources. The interbeam interference between the beams must be considered in the power allocation; therefore, it is important to optimize the power allocated to each beam in order to improve the total system performance. Initially the power allocation problem is formulated as a nonlinear optimization, considering a compromise between the maximization of total system capacity and the fairness of the power allocation amongst the beams. A dynamic power allocation algorithm based on duality theory is then proposed to obtain a locally optimal solution for the optimization problem. Compared with traditional power allocation algorithms, this proposed dynamic power allocation algorithm improves the fairness of the power allocation amongst the beams, and, in addition, the proposed algorithm also increases the total system capacity in certain scenarios.
\end{abstract}

\section{Introduction}

In satellite communication systems, a satellite may provide coverage of the entire region of the earth visible from the satellite, by using a single beam. In this case, the gain of the satellite antenna will be limited by the beamwidth, as imposed by the coverage. For instance, for a geostationary satellite, global coverage implies a $3 \mathrm{~dB}$ beamwidth of 17.5 and consequently an antenna gain of no more than $20 \mathrm{~dB}$ [1]. Therefore, each user must be equipped with a large aperture antenna to support the high traffic rate, which results in great inconvenience. In order to solve this problem, the multibeam technique has been widely applied in modern satellite communication systems. In multibeam satellite communication systems, the satellite provides coverage of only part of the earth, by means of a narrow beam. The benefit of a higher satellite antenna gain is obtained due to a reduction in the aperture angle of the antenna beam [1]. As a result, a user with a small aperture antenna can support a high traffic rate. Moreover, the multibeam technique supports the reuse of frequencies for different beams, in order to increase the total system capacity. When two beams utilize the same frequency, interbeam interference is introduced to the two beams, due to the nonzero gain of the antenna side lobe. It has been noted that when there is interbeam interference between the beams, the capacity allocated to each beam is determined not only by the power allocated to the beam, but also by the power allocated to the other beams.

Due to the limitations of satellite platform, it is known that satellite power resources are scarce and expensive. It is thus important to optimize the utilization efficiency of the power resources. Moreover, the traffic demands of each beam are different, with varying times, due to the different coverage areas, and the interbeam interference between the different beams is also different. As a result, it is critical to optimize the power allocation to each beam to meet the specific traffic demands.

Power allocation algorithms were proposed in earlier works [2-7]. The mathematical formulation and analytic solutions of the optimum power allocation problem have been presented [2]; however, the mathematical algorithm to solve the optimization problem was not provided. As a result, bisection and subgradient methodologies have been utilized to solve the optimization problem $[3,4]$. In order to improve the total system capacity, a method to select a small number of active beams has been proposed [5], which maintained the fairness of the power allocation amongst the 
beams. The main problem in [2-5] was that the authors failed to consider the interbeam interference between the beams, which cannot be ignored in determining power allocations. A novel resource allocation scheme for multibeam satellite communication systems has been described, offered maximum communication capacity [6]. The scheme optimized the frequency bandwidth, the satellite transmission power, the modulation level, and the coding rate to each beam, in order to manage the ever-changing user distributions and the interbeam interference conditions. However, the scheme ignored the fairness of the power allocations amongst the beams. A joint optimization allocation algorithm for the power and the carrier was proposed [7], in order to best match the asymmetric traffic requests. The algorithm attempted to support the greatest degree of fairness in the power allocation to each beam, regardless of the total system capacity.

This paper's research is aimed at resolving this deficiency, by optimizing the power allocations for a multibeam satellite communication system, with full consideration of the impact of interbeam interference. The first step is to mathematically formulate the problem of power allocation as a non-linear optimization, compromising between the maximization of total system capacity and the fairness of the power allocations to each beam. It is found that, in the optimization process, the optimal variables are coupled with each other. As a result, it is difficult to determine whether the optimization is convex or not and to obtain the globally optimal solution for the optimization. To this end, a dynamic power allocation algorithm based on duality theory is proposed to obtain a locally optimal solution for the optimization. Finally, the simulation results show the efficiency of the proposed dynamic power allocation algorithm.

The main contributions of this research are summarized as follows:

(1) the mathematical formulation of the power allocation problem for multibeam satellite communication, with consideration of interbeam interference, through a compromise between the maximization of the total system capacity and the fairness of the power allocation amongst the beams;

(2) the proposal of an algorithm, based on duality theory, which will obtain a locally optimal solution for the optimization problem;

(3) a demonstration of the effects of the interbeam interference and the channel conditions of each beam on the power allocation results.

The remainder of this paper is organized as follows. In Section 2, the model of a multibeam satellite communication system with interbeam interference is described. In Section 3, a mathematical formulation of the optimization problem of the power allocation is presented. Section 4 presents the proposal of a dynamic power allocation algorithm designed to obtain a locally optimal solution to the optimization. Section 5 presents the simulation results and analyzes the effects of the interbeam interference and channel conditions

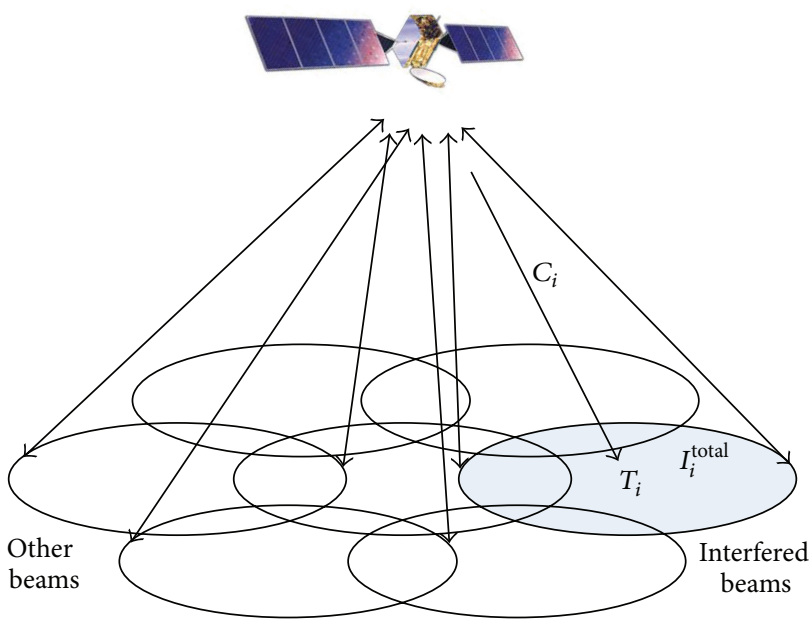

FIGURE 1: Configuration of the multibeam satellite communication system.

of each beam on the power allocation results. Section 6 presents the conclusion of the paper.

\section{A Multibeam Satellite Communication System Model}

Figure 1 shows the system configuration of the multibeam satellite communication system that is studied here, where

$N$ : is the quantity of the beams,

$T_{i}$ is the traffic demand of the $i$ th beam,

$P_{i}$ is the power allocated to the $i$ th beam,

$I_{i}^{\text {total }}$ is the total interference on the $i$ th beam from the other beams,

$\gamma$ is the signal attenuation factor of the $i$ th beam, and it is noted that $\gamma$ mainly consisted of the effects of weather conditions, free space loss, and antenna gain, and

$P_{\text {total }}$ is the total satellite power resources within the system.

To precisely describe the interbeam interference within the system, the interbeam interference matrix $H$ is introduced, which is defined as follows:

$$
H=\left[\begin{array}{cccc}
0 & h_{12} & \cdots & h_{1 n} \\
h_{21} & 0 & \cdots & h_{2 n} \\
\vdots & \vdots & \ddots & \vdots \\
h_{n 1} & h_{n 2} & \cdots & 0
\end{array}\right],
$$

where the element $h_{i j}$ denotes the interference coefficient from the $j$ th beam on the $i$ th beam. It is noted that the element $h_{i i}$ is zero, because the interference from the same beam is ignored. It is obvious from (1) that the total interference on the 
$i$ th beam from other beams $I_{i}^{\text {total }}$ is $\sum_{k=1, k \neq i}^{N} P_{k} h_{i, k}$. As a result, using time sharing for Gaussian broadcast channels [8], the Shannon bounded capacity $C_{i}$ for the $i$ th beam is given as

$$
C_{i}=W \log _{2}\left(1+\frac{P_{i}}{\gamma W N_{0}+\sum_{k=1, k \neq i}^{N} P_{k} h_{i, k}}\right)
$$

where $N_{0}$ is the noise power density of each beam and $W$ is the bandwidth of each beam. It is shown in (2) that the capacity $C_{i}$ of the $i$ th beam is increased as the power allocated to the beam increases. However, the capacity is decreased as the power allocated to other beams increases, due to the interbeam interference. As a result, the capacity of each beam is determined not only by the power allocated to it, but also by the power allocated to the other beams.

\section{Mathematical Formulation of the Power Allocation}

In this paper, the metric to evaluate the power allocation results minimizes the sum of the squared differences between the traffic demand and the capacity allocated to each beam. As a result, the metric will ensure a relatively greater capacity allocation to the beams when there are higher traffic demands, which will achieve greater fairness of the power allocations amongst the beams. At the same time, the metric will also work to maximize the total system capacity. Therefore, the metric considers a compromise between the maximization of total system capacity and the fairness of the power allocations amongst the beams. As a result, the optimization is formulated as follows:

$$
\begin{array}{cc}
\min _{\left\{P_{i}\right\}} & \sum_{i=1}^{N}\left(T_{i}-C_{i}\right)^{2} \\
\text { s.t. } & C_{i}=W \log _{2}\left(1+\frac{P_{i}}{\gamma W N_{0}+\sum_{k=1, k \neq i}^{N} P_{k} h_{i, k}}\right), \\
& \sum_{i=1}^{N} P_{i} \leq P_{\text {total }} .
\end{array}
$$

When there is no interbeam interference between the beams, each of the elements in the interbeam interference matrix is equal to zero. As a result, the optimization is convex [2], and the globally optimal solution can be obtained by the optimization. However, when interbeam interference actually exists, it is seen that the optimal variables $P_{i}$ are coupled with each other. Therefore, it is difficult to determine whether the optimization is convex or not and to obtain the globally optimal power solution for the optimization. To this end, an algorithm based on duality theory is proposed to obtain a locally optimal solution for the optimization [9-12], as presented in the following section.

\section{Proposed Dynamic Power Allocation Algorithm}

As mentioned above, the proposed dynamic power allocation algorithm is based on duality theory [13]. By introducing the nonnegative dual variable $\lambda$, the Lagrange function is given by

$$
L(\mathbf{P}, \lambda)=\sum_{i=1}^{N}\left(T_{i}-C_{i}\right)^{2}-\lambda\left(P_{\text {total }}-\sum_{i=1}^{N} P_{i}\right),
$$

where $\mathbf{P}=\left[P_{1}, P_{2}, \ldots, P_{N}\right]$.

From (4), the Lagrange dual function can be obtained by

$$
D(\lambda)=\min _{\mathbf{P}} L(\mathbf{P}, \lambda)
$$

and the dual problem can be written as

$$
d^{*}=\max _{\lambda \geq 0, \sigma_{i} \geq 0} D(\lambda)
$$

The dual problem in (6) can be further decomposed into the following two sequentially iterative subproblems [9].

Subproblem 1: Power Allocation. Given the dual variable $\lambda$, for any: $i \in\{1,2, \ldots, N\}$, differentiating (4) with respect to $P_{i}$ results in the equation below:

$$
\frac{\partial D(\boldsymbol{\sigma}, \lambda)}{\partial P_{i}}=2 \sum_{j=1}^{N}\left(T_{j}-C_{j}\right) \frac{\partial C_{j}}{\partial P_{i}}-\lambda=0 .
$$

The optimized power allocation of the $i$ th beam $P_{i}$ can be obtained in (7) by numerical calculation methods, for example, the golden section. Moreover, if the optimized $P_{i}$ is less than zero, then $P_{i}$ is set to be zero. The detailed expressions in (7) are shown in the appendix.

Subproblem 2: Dual Variable Update. The optimal dual variable can be obtained by solving the dual problem:

$$
\lambda^{\mathrm{opt}}=\arg \max _{\lambda} \min \left[L\left(\mathbf{P}^{\mathrm{opt}}, \lambda\right)\right] .
$$

Because the dual function is always convex, a subgradient method (a generalization of the gradient) can be used here to update the dual variable, as shown below [9]:

$$
\lambda^{n+1}=\left[\lambda^{n}-\Delta_{\lambda}^{n}\left(P_{\text {total }}-\sum_{i=1}^{N} P_{i}^{\mathrm{opt}}\right)\right]^{+},
$$

where $[x]^{+}=\max \{0, x\}, n$ is the iteration number, and $\Delta$ is the iteration step size.

It has been proven that the above dual variable updating algorithm is guaranteed to converge to the optimal solution, as long as the iteration step chosen is sufficiently small [9].

The whole process of the proposed dynamic power allocation algorithm is summarized as follows.

Step 1. Set appropriate values to $\lambda$ and $P_{i}, i \in\{1,2, \ldots, N\}$.

Step 2. Calculate the value of $P_{i}$ from (7). 
Step 3. Substitute the power values of each beam, as obtained from Step 2, into (9) and then update the dual variable.

Step 4. If the condition of $\left|\lambda^{n+1}\left(P_{\text {total }}-\sum_{i=1}^{N} P_{i}\right)\right|<\varepsilon$ is satisfied, then terminate the algorithm; otherwise, jump to Step 2.

Utilizing the above process, the allocated power to each beam is obtained.

\section{Simulation Results and Analysis}

For the simulation, a multibeam satellite communication system model is set up. The system has 10 beams. For each beam, the bandwidth resource is $50 \mathrm{MHz}$ and the normalized noisepower spectral density parameter $\gamma N_{0}$ is $0.2 e^{-6}$. Total satellite power is $200 \mathrm{~W}$. The traffic demand of each beam is increased from $80 \mathrm{Mbps}$ to $170 \mathrm{Mbps}$, by steps of $10 \mathrm{Mbps}$.

5.1. The Efficiency of the Proposed Power Allocation Algorithm. In order to show the efficiency of the proposed dynamic power allocation algorithm, it is compared with the following two traditional algorithms.

(1) Uniform power allocation algorithm: $P_{i}=P_{\text {total }} / N$.

(2) Proportional power allocation algorithm: $P_{i}=$ $P_{\text {total }} T_{i} / T_{\text {total }}$, where $T_{\text {total }}$ is the total traffic demand of all the beams.

Moreover, comparisons are made of the power allocation results for the three algorithms in the following two scenarios, with different interbeam interference matrixes.

Scenario 1. In this system, each beam interferes with the three adjacent beams. As a result, the element in the interbeam interference matrix is set as follows:

$$
h_{i j}= \begin{cases}0.3, & \text { if }|j-i|=1 \text { or }|j-i \pm 10|=1 \\ 0.2, & \text { if }|j-i|=2 \text { or }|j-i \pm 10|=2 \\ 0.1, & \text { if }|j-i|=3 \text { or }|j-i \pm 10|=3 \\ 0, & \text { esle. }\end{cases}
$$

Figure 2 shows the capacity allocated to each beam for the three power allocation algorithms in Scenario 1. Table 1 presents the total system capacities of the three power allocation algorithms in Scenario 1. As shown in Figure 2, the uniform power allocation algorithm uniformly allocates power to each beam, regardless of the traffic demand of the beams or the fairness of the power allocations amongst the beams. Moreover, the total interference from the other beams is the same for each beam, and as a result the capacity allocated to each beam is the same. The proportional power allocation algorithm allocates the power resources to each beam solely according to the traffic demand of each beam, regardless of the interbeam interference. Therefore, the capacity allocated to a beam with high traffic demand is higher than that allocated to a beam with low traffic demand. Compared with the proportional power allocation algorithm, the proposed dynamic power allocation algorithm

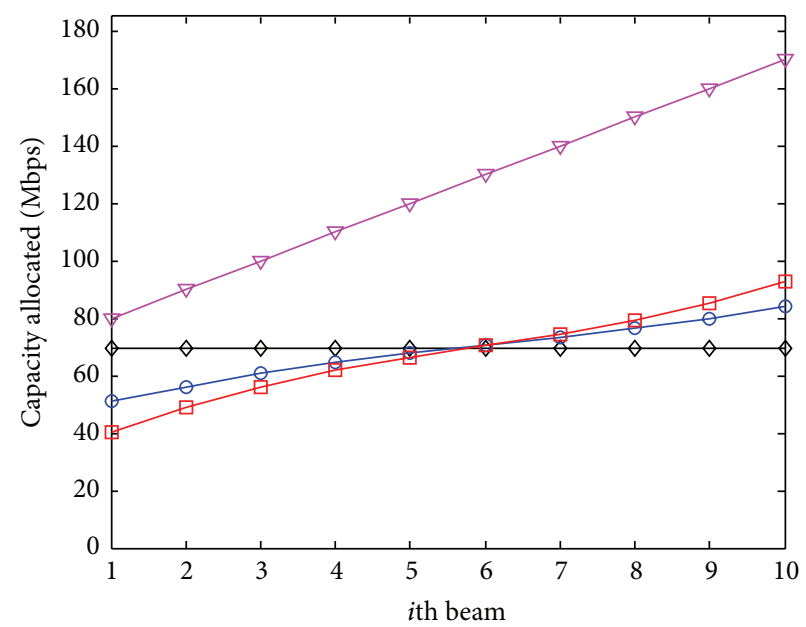

$\begin{array}{ll}\nabla \text { Traffic demand } & \odot \text { Proportional power allocation } \\ \neg \text { Uniform power allocation } & \square-\text { Proposed dynamic power } \\ & \text { allocation }\end{array}$

FIGURE 2: Distribution of the capacity allocated to each beam, for the three algorithms in Scenario 1.

TABLE 1: Total system capacity of the three algorithms in Scenario 1.

\begin{tabular}{lc}
\hline Algorithm & $\sum C_{i}$ \\
\hline Uniform power allocation & $692.83(\mathrm{Mbps})$ \\
Proportional power allocation & $685.20(\mathrm{Mbps})$ \\
Proposed dynamic power allocation & $675.03(\mathrm{Mbps})$ \\
\hline
\end{tabular}

TABLE 2: System's total squared difference for the three algorithms from Scenario 1.

\begin{tabular}{lc}
\hline Algorithm & $\sum\left(T_{i}-C_{i}\right)^{2}$ \\
\hline Uniform power allocation & $3.93 E 16$ \\
Proportional power allocation & $3.54 E 16$ \\
Proposed dynamic power allocation & $3.48 E 16$ \\
\hline
\end{tabular}

allocates more power resources to beams having higher traffic demands, in order to minimize the system's total squared difference between the traffic demand and the capacity allocated to each beam. However, due to the concavity of the capacity function in terms of allocated power, the total system capacity is decreased, which is also shown by the data in Table 1.

Figure 3 shows the squared difference between the traffic demand and allocated capacity of each beam, for the three algorithms from Scenario 1. Table 2 presents the total squared difference for the three algorithms in Scenario 1. As mentioned above, the proposed dynamic power allocation algorithm provides more power resources to the beams with higher traffic demands. As a result, when the results of the proposed dynamic power allocation algorithm are compared to the results of the other two algorithms, the squared difference for the beams with high traffic demands is lower and the squared difference for the beams with low traffic demand is higher. Moreover, the total squared difference for the proposed dynamic power algorithm is the lowest of the 


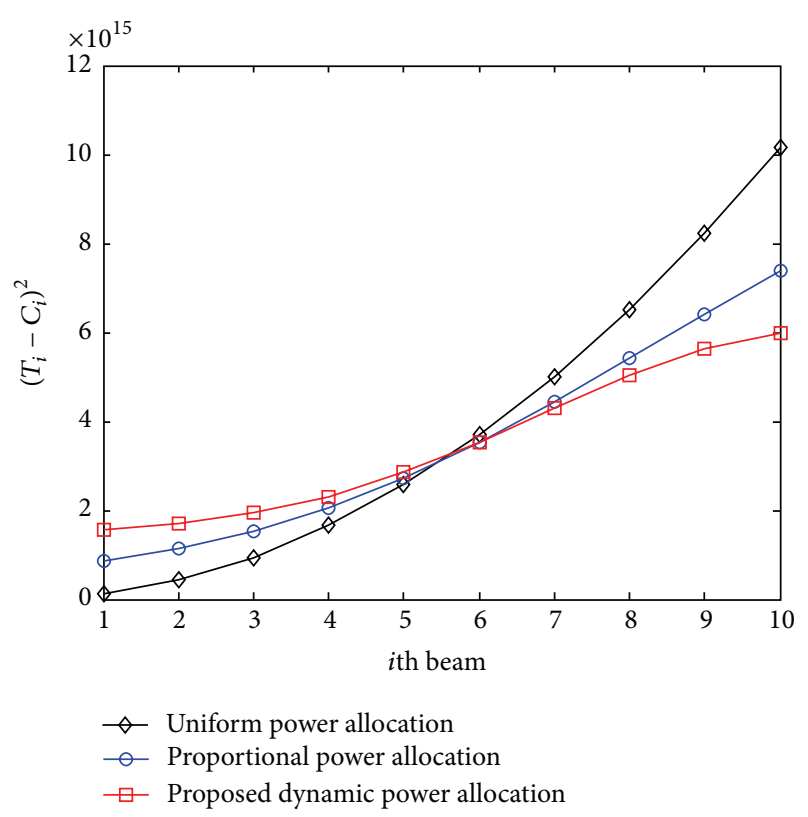

Figure 3: Distribution of the squared difference between the traffic demand and the allocated capacity of each beam, for each of the three algorithms from Scenario 1.

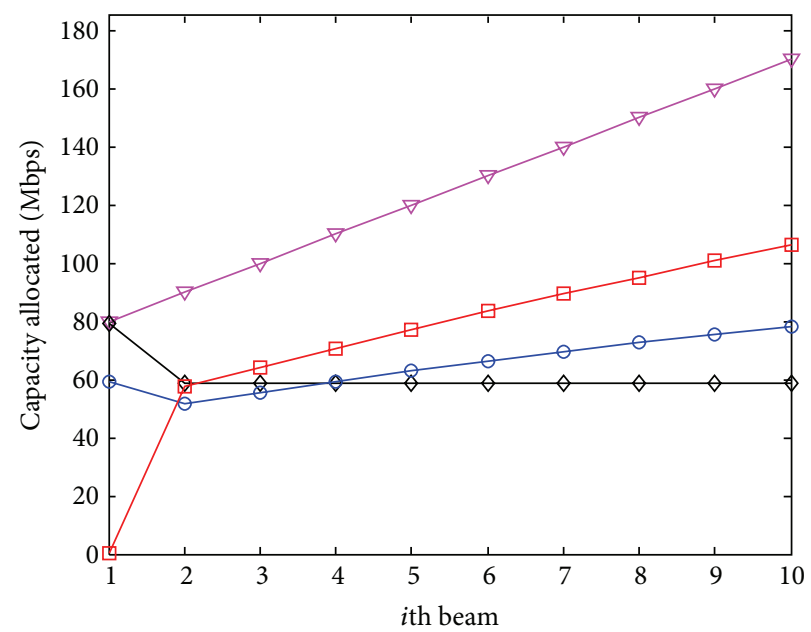

$$
\begin{array}{ll}
\neg \text { Traffic demand } & - \text { Proportional power allocation } \\
\neg \text { Uniform power allocation } & \square-\text { Proposed dynamic power } \\
& \text { allocation }
\end{array}
$$

FIGURE 4: Distribution of the allocated capacity to each beam, for the three algorithms in Scenario 2.

three algorithms. This conclusion is also demonstrated by the data shown in Table 2 .

Scenario 2. In this scenario, it is assumed that there is a hostile interference source in Beam 1. Thus we consider that only Beam 1 interferes with the other beams. The interbeam interference matrix is set as follows:

$$
h_{i j}= \begin{cases}0.3, & i=\{2, \ldots 10\}, j=1 \\ 0, & \text { else. }\end{cases}
$$

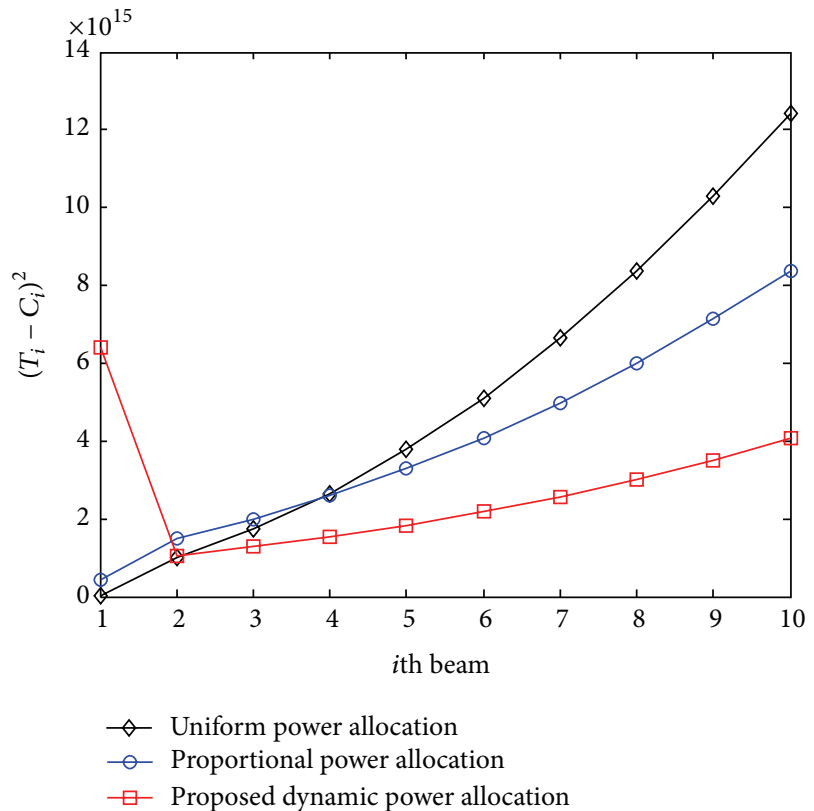

FIGURE 5: Distribution of the squared difference between the traffic demand and the capacity allocated to each beam, for the three algorithms in Scenario 2.

Figure 4 shows the allocated capacity for each beam, for the three power allocation algorithms in Scenario 2. Table 3 presents the total system capacities of the three power allocation algorithms from Scenario 2. It is known that only Beam 1 interferes with the other beams. It will seem reasonable that, by allocating less power to Beam 1 , the interference on the other beams will decrease, and the total system capacity will increase. To this end, the proposed dynamic power allocation algorithm allocates no power to Beam 1 to decrease its interference with the other beams. However, both the uniform and the proportional power allocation algorithms allocate power to each beam, regardless of the interbeam interference matrix in the system. Thus, the power allocated to each beam in Scenario 2 is the same as that in Scenario 1, and the power allocated to Beam 1 is not decreased. As a result, the total system capacity obtained by the two algorithms is less than that obtained by the proposed dynamic power allocation algorithm, as shown in Table 3.

Figure 5 shows the squared difference between the traffic demand and the capacity allocated to each beam, for the three algorithms in Scenario 2. Table 4 presents the total squared difference for the three algorithms in Scenario 2. Figure 5 shows that the squared difference of Beam 1 obtained by the proposed algorithm is higher than that obtained by the other two algorithms. However, the squared differences from Beams 2 to 10 are lower. This is because when compared with the other two algorithms, the proposed dynamic power allocation algorithm provides no power resources to Beam 1 and provides more power to Beams 2 through 10. Moreover, the total squared difference of the proposed dynamic power allocation algorithm is less than that of the other two power allocation algorithms. Taken together with the conclusion 
TABLE 3: Total system capacity for the three algorithms in Scenario 2 .

\begin{tabular}{lc}
\hline Algorithm & $\sum C_{i}$ \\
\hline Uniform power allocation & $605.71(\mathrm{Mbps})$ \\
Proportional power allocation & $650.39(\mathrm{Mbps})$ \\
Proposed dynamic power allocation & $744.40(\mathrm{Mbps})$ \\
\hline
\end{tabular}

TABLE 4: System's total squared difference for the three algorithms in Scenario 2.

\begin{tabular}{lc}
\hline Algorithm & $\sum\left(T_{i}-C_{i}\right)^{2}$ \\
\hline Uniform power allocation & $5.20 E 16$ \\
Proportional power allocation & $3.54 E 16$ \\
Proposed dynamic power allocation & $2.75 E 16$ \\
\hline
\end{tabular}

TABLE 5: Total system capacity for the three algorithms in Scenario 3.

\begin{tabular}{lc}
\hline Algorithm & $\sum C_{i}$ \\
\hline Uniform power allocation & $519.29(\mathrm{Mbps})$ \\
Proportional power allocation & $560.92(\mathrm{Mbps})$ \\
Proposed dynamic power allocation & $655.70(\mathrm{Mbps})$ \\
\hline
\end{tabular}

about the total system capacity, it is clear that the proposed dynamic power allocation algorithm improves both the system capacity and the fairness of the power allocations amongst the beams in this scenario.

It is noted that the traffic demand and the channel conditions of each beam are the same in the two scenarios, and only the interbeam interference matrix is different. However, the power allocation result obtained by the proposed algorithm shows a great difference in the two scenarios. In other words, the interbeam interference between the beams has a significant impact on the power allocation results. In addition, the proposed algorithm dynamically allocates the power resource to each beam, taking into account the impact of the interbeam interference between the beams, making the best effort in removing the adverse impacts of the interbeam interference.

\subsection{The Effects of the Channel Condition of Each Beam on the} Power Allocation Results. It is known that signal attenuation factor $\gamma$ is affected by channel conditions. To show the impact of the channel conditions of each beam on the power allocation results, the following scenario is set up.

Scenario 3. The normalized noise power spectral density parameters $\gamma N_{0}$ from Beams 3 through 5 are set to be $0.2 e^{-6}$, $1.2 e^{-6}$, and $2.2 e^{-6}$. The traffic demand of the three beams is set to be the same as $100 \mathrm{Mbps}$. The interbeam interference matrix is set to be the same as that in Scenario 2, and other parameters in the system remained the same.

Figure 6 shows the capacity allocated to each beam for the three power allocation algorithms when the channel conditions of each beam are different. Table 5 presents the total system capacity for the three power allocation algorithms

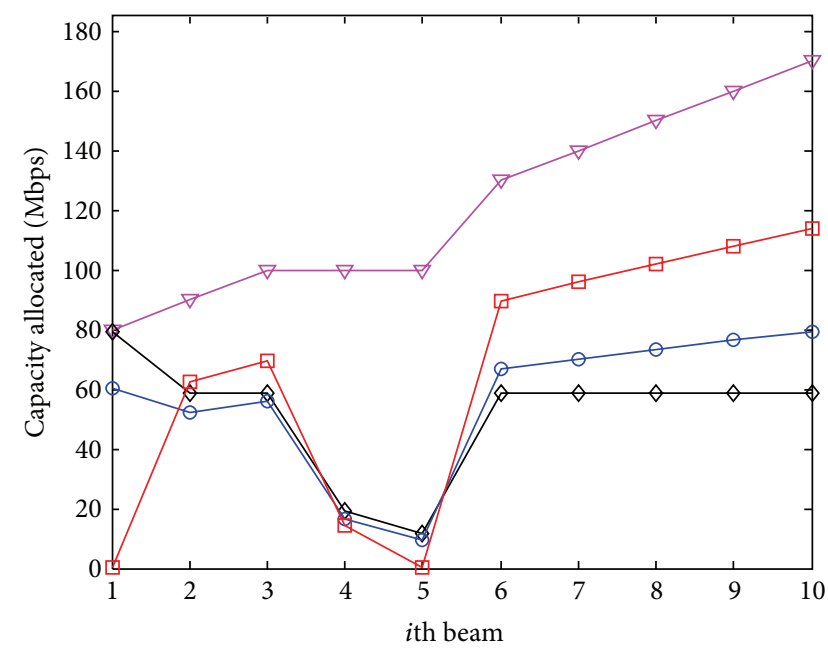

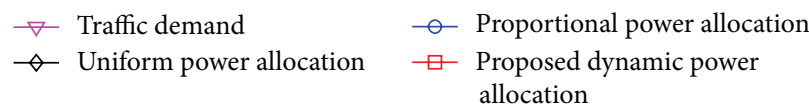

FIGURE 6: Distribution of the capacity allocated to each beam, for the three algorithms in Scenario 3.

TABLE 6: System's total squared difference for the three algorithms in Scenario 3.

\begin{tabular}{lc}
\hline Algorithm & $\sum\left(T_{i}-C_{i}\right)^{2}$ \\
\hline Uniform power allocation & $5.99 E 16$ \\
Proportional power allocation & $4.88 E 16$ \\
Proposed dynamic power allocation & $3.71 E 16$ \\
\hline
\end{tabular}

in Scenario 3. It is noted that the traffic demand and total interference from the other beams, for Beams 3 through 5 , are the same, and only the channel conditions of the three beams are different. As shown in Figure 6, the proposed dynamic power allocation algorithm provides more power resources to the beams that have better channel conditions, and rarely or never provides power to beams with worse channel conditions. Beam 5, for example, with the worst channel condition is provided with no power resources. Therefore, the proposed dynamic power allocation algorithm not only considers the fairness of the power allocations amongst the beams, but also tries to maximize the throughput of the system and achieves a good system performance as predicted. The proportional or uniform power allocation algorithms cannot dynamically allocate the power resources to each beam according to their channel conditions; thus their total system capacities are less than that of the proposed dynamic power allocation algorithm, as clearly demonstrated by the data shown in Table 5 .

Figure 7 shows the squared difference between the traffic demand and the capacity allocated to each beam, for the three algorithms in Scenario 3. Table 6 presents the total squared difference of the three algorithms in Scenario 3. As mentioned above, the proposed dynamic power allocation algorithm provides more power resources to the beams having better channel conditions. As a result, the squared difference of the 


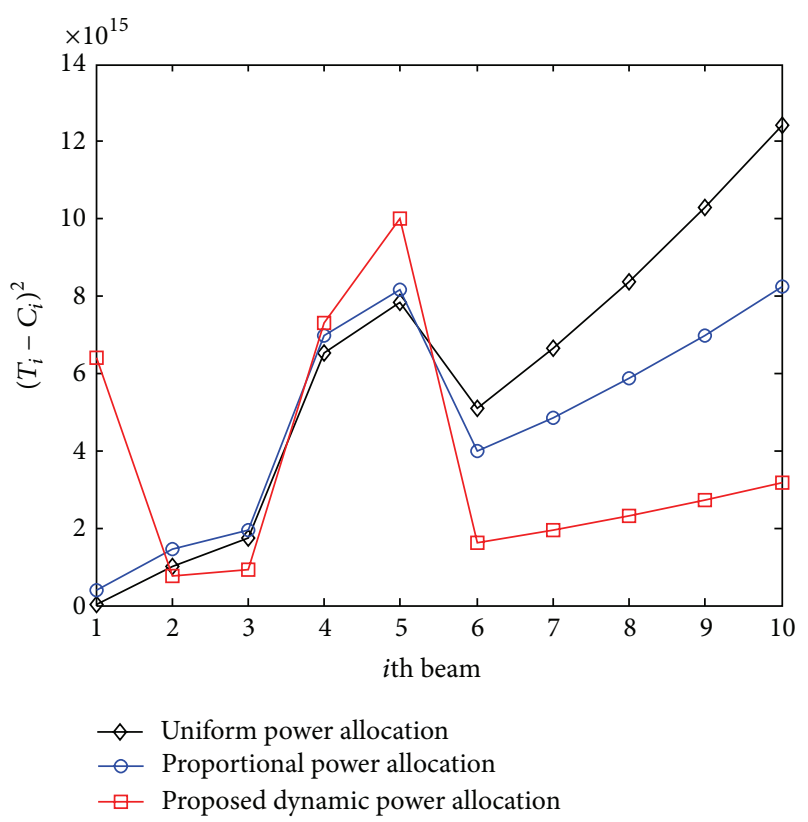

Figure 7: Distribution of squared difference between the traffic demand and the capacities allocated to each beam, for the three algorithms in Scenario 3.

traffic demand and the capacity allocated to the beams with better channel conditions is smaller than that of the other two algorithms, as shown in Figure 7. Moreover, the total squared difference of the proposed dynamic power allocation algorithm is also the smallest of the three algorithms. Thus, the fairness of the power allocations amongst the beams that is obtained by the proposed dynamic power allocation algorithm provides the optimal optimization.

\section{Conclusions}

In multibeam satellite communication systems, due to the reusing of frequencies, there exists interbeam interference between the beams, which cannot be ignored in determining power allocations. To precisely describe the impact of the interbeam interference, the problem of power allocation as a non-linear optimization with constraints was formulated, including a compromise between the maximization of total system capacity and the fairness of the power allocations to the beams. A dynamic power allocation algorithm was then proposed to obtain a locally optimal solution to the optimization.

It was shown that, compared with the traditional uniform or proportional power allocation algorithms, the proposed dynamic power allocation algorithm improved the fairness of the power allocations to the beams and also increased the total system capacity in certain scenarios, such as Scenarios 2 and 3 as presented in Section 5. In addition, the interbeam interference between both the beams and the channel conditions of each beam had a significant impact on the power allocation results. The proposed dynamic power allocation algorithm functioned to remove the adverse impacts of these factors; for example, the algorithm allocated less power to the beams which had greater interference on the other beams, or which had worse channel conditions.

\section{Appendix}

Equation (7) is expressed in detail.

When $i=j, \partial C_{j} / \partial P_{i}$ is given as

$$
\frac{\partial C_{i}}{\partial P_{i}}=\frac{W}{\ln 2} \cdot \frac{1}{\gamma W N_{0}+\sum_{k=1, k \neq i}^{N} P_{k} h_{i k}+P_{i}} .
$$

When $i \neq j, \partial C_{j} / \partial P_{i}$ is expressed as

$$
\begin{aligned}
\frac{\partial C_{j}}{\partial P_{i}}= & \frac{-W}{\ln 2} \\
& \cdot P_{j} h_{j i} \times\left(\left(\gamma W N_{0}+\sum_{k=1, k \neq j}^{N} P_{k} h_{j k}\right)^{2}\right. \\
& \left.+P_{j}\left(\gamma W N_{0}+\sum_{k=1, k \neq j}^{N} P_{k} h_{j k}\right)\right)^{-1} .
\end{aligned}
$$

Substituting (A.1) and (A.2) into (7), the following equation is obtained:

$$
\begin{aligned}
\left(T_{i}\right. & \left.-C_{i}\right) \frac{2 W}{\ln 2} \cdot \frac{1}{\gamma W N_{0}+\sum_{k=1, k \neq i}^{N} P_{k} h_{i k}+P_{i}}-\lambda \\
= & \sum_{j=1, j \neq i}^{N} \frac{W}{\ln 2}\left(2 T_{j}-2 C_{j}\right) \cdot M_{j i},
\end{aligned}
$$

where

$$
\begin{aligned}
M_{j i}=P_{j} h_{j i} \times\left(\left(\gamma W N_{0}+\sum_{k=1, k \neq j}^{N} P_{k} h_{j k}\right)^{2}\right. & \\
& \left.+P_{j}\left(\gamma W N_{0}+\sum_{k=1, k \neq j}^{N} P_{k} h_{j k}\right)\right)^{-1} .
\end{aligned}
$$

According to (A.3), the optimized power allocation of the $i$ th beam $P_{i}$ could be obtained by numerical calculation methods.

\section{Conflict of Interests}

The authors declare that they do not have any commercial or associative interests that represents a conflict of interest in connection with the work submitted.

\section{Acknowledgment}

The authors would like to thank the project support provided by the National High-Tech Research \& Development Program of China under Grant 2012AA01A508. 


\section{References}

[1] G. Maral, M. Bousquet, and Z. Sun, Communications Systems: Systems, Techniques and Technology, John Wiley and Sons, New York, NY, USA, 5th edition, 2009.

[2] J. P. Choi and V. W. S. Chan, "Optimum power and beam allocation based on traffic demands and channel conditions over satellite downlinks," IEEE Transactions on Wireless Communications, vol. 4, no. 6, pp. 2983-2992, 2005.

[3] Y. Hong, A. Srinivasan, B. Cheng, L. Hartman, and P. Andreadis, "Optimal power allocation for multiple beam satellite systems," in Proceedings of the IEEE Radio and Wireless Symposium (RWS '08), pp. 823-826, January 2008.

[4] Q. Feng, G. Li, S. Feng, and Q. Gao, "Optimum power allocation based on traffic demand for multi-beam satellite communication systems," in Proceedings of the 13th International Conference on Communication Technology (ICCT '11), pp. 873-876, September 2011.

[5] U. Park, H. W. Kim, D. S. Oh, and B. J. Ku, "Optimum selective beam allocation scheme for satellite network with multi-spot beams," in Proceedings of the 4th International Conference on Advances in Satellite and Space Communications (SPACOMM '12), pp. 78-81, 2012.

[6] K. Nakahira, K. Kobayashi, and M. Ueba, "Capacity and quality enhancement using an adaptive resource allocation for multibeam mobile satellite communication systems," in Proceedings of the IEEE Wireless Communications and Networking Conference (WCNC '06), pp. 153-158, April 2006.

[7] J. Lei and M. A. Vázquez-Castro, "Joint power and carrier allocation for the multibeam satellite downlink with individual SINR constraints," in Proceedings of the IEEE International Conference on Communications (ICC '10), May 2010.

[8] T. M. Cover and J. A. Thomas, Elements of Information Theory, John Wiley \& Sons, New York, NY, USA, 1991.

[9] W. Yu and R. Lui, "Dual methods for nonconvex spectrum optimization of multicarrier systems," IEEE Transactions on Communications, vol. 54, no. 7, pp. 1310-1322, 2006.

[10] R. Wang, V. K. N. Lau, L. Lv, and B. Chen, "Joint cross-layer scheduling and spectrum sensing for OFDMA cognitive radio systems," IEEE Transactions on Wireless Communications, vol. 8, no. 5, pp. 2410-2416, 2009.

[11] G. Ding, Q. Wu, and J. Wang, "Sensing confidence levelbased joint spectrum and power allocation in cognitive radio networks," Wireless Personal Communications, vol. 72, no. 1, pp. 283-298, 2013.

[12] A. G. Marques, X. Wang, and G. B. Giannakis, "Dynamic resource management for cognitive radios using limited-rate feedback," IEEE Transactions on Signal Processing, vol. 57, no. 9, pp. 3651-3666, 2009.

[13] S. Boyd and L. Vandenberghe, Convex Optimization, Cambridge University Press, Cambridge, UK, 2004. 


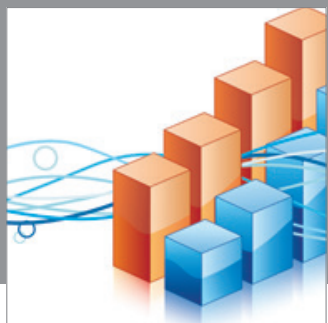

Advances in

Operations Research

mansans

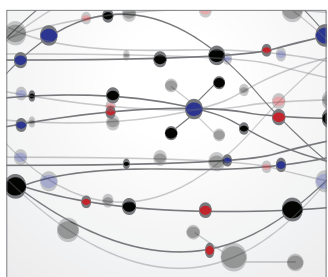

The Scientific World Journal
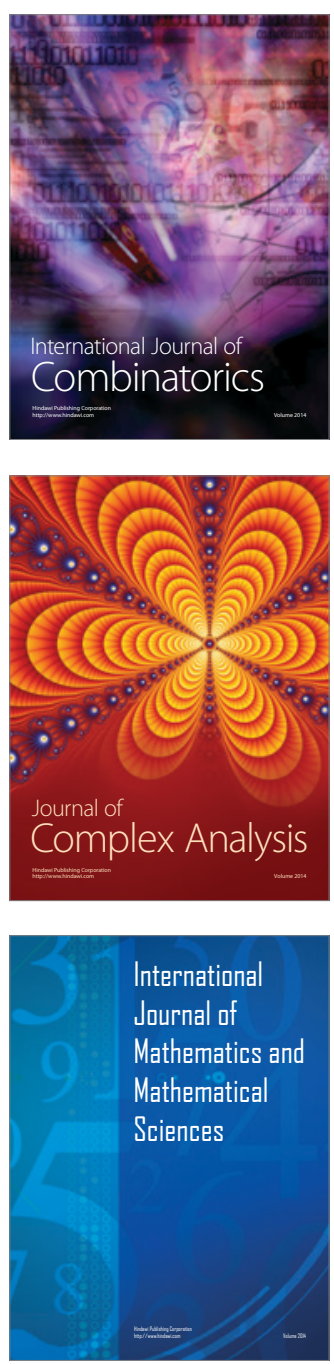
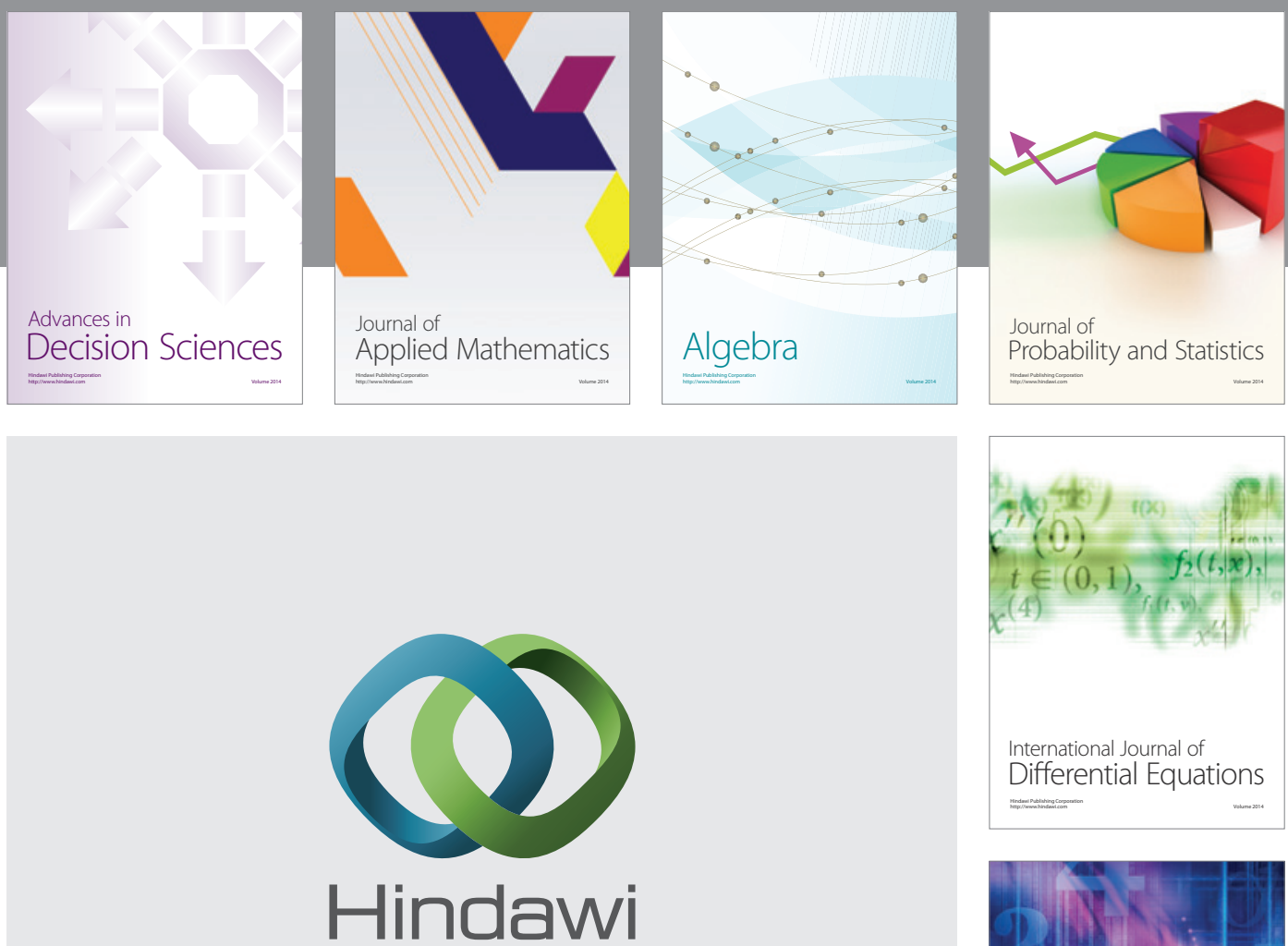

Submit your manuscripts at http://www.hindawi.com
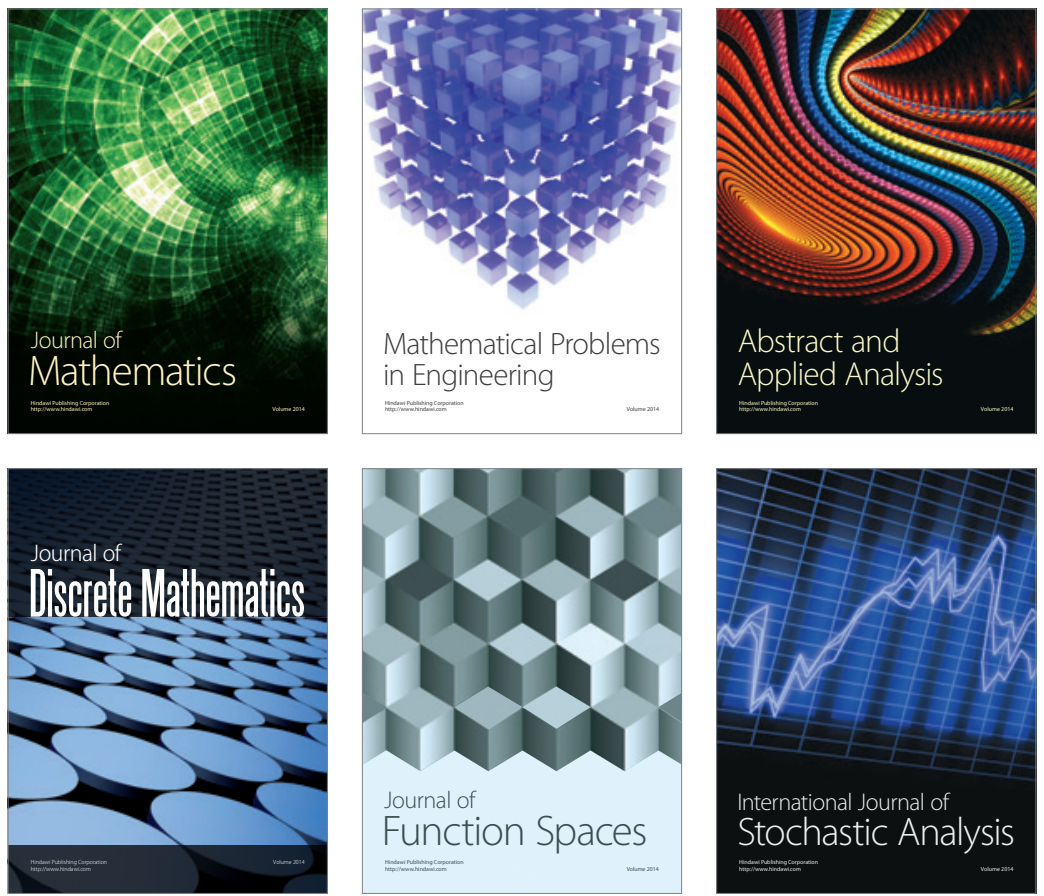

Journal of

Function Spaces

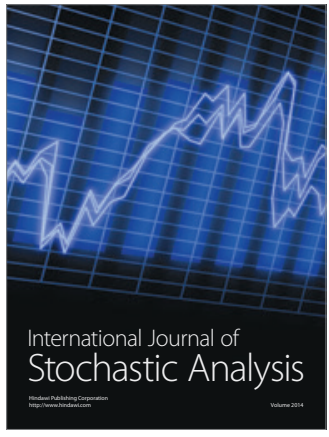

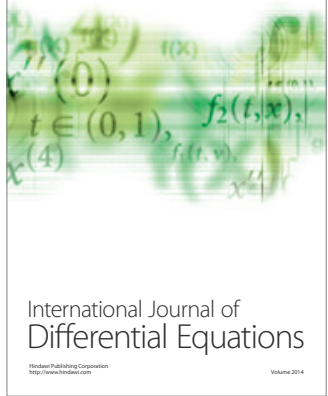
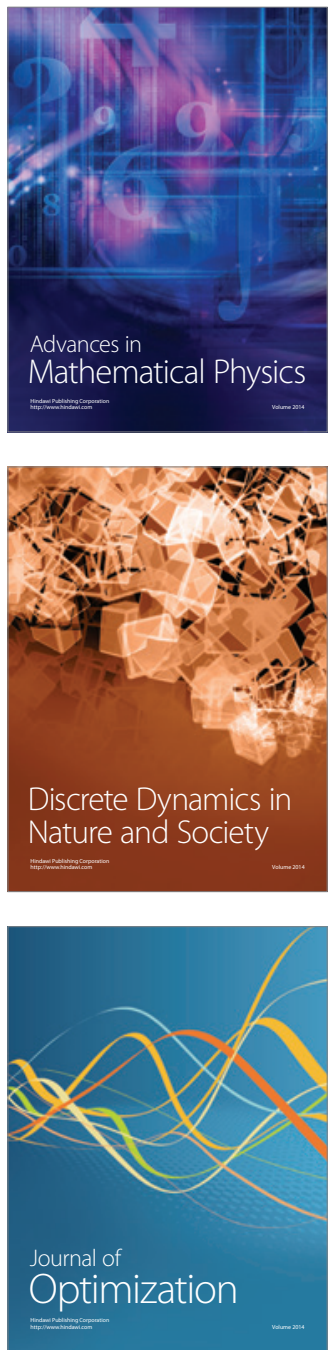